\title{
Selective randomized load balancing and mesh networks with changing demands
}

\author{
F. B. Shepherd and P. J. Winzer \\ Fundamental Mathematics Research, Data/Optical Networks Research, Bell Labs \\ ReCEIVED 21 NOVEMber 2005; REVISED 6 MARCH 2006; \\ ACCEPTED 8 MARCH 2006; PUBLISHED 5 APRIL 2006 \\ We consider the problem of building cost-effective networks that are robust to \\ dynamic changes in demand patterns. We compare several architectures using \\ demand-oblivious routing strategies. Traditional approaches include single-hop \\ architectures based on a (static or dynamic) circuit-switched core infrastructure \\ and multihop (packet-switched) architectures based on point-to-point circuits in \\ the core. To address demand uncertainty, we seek minimum cost networks that \\ can carry the class of hose demand matrices. Apart from shortest-path routing, \\ Valiant's randomized load balancing (RLB), and virtual private network (VPN) \\ tree routing, we propose a third, highly attractive approach: selective randomized \\ load balancing (SRLB). This is a blend of dual-hop hub routing and randomized \\ load balancing that combines the advantages of both architectures in terms of \\ network cost, delay, and delay jitter. In particular, we give empirical analyses \\ for the cost (in terms of transport and switching equipment) for the discussed \\ architectures, based on three representative carrier networks. Of these three \\ networks, SRLB maintains the resilience properties of RLB while achieving \\ significant cost reduction over all other architectures, including RLB and \\ multihop Internet protocol/multiprotocol label switching (IP/MPLS) networks \\ using VPN-tree routing. (C) 2006 Optical Society of America
}

OCIS codes: $060.4250,000.5490$.

\section{Introduction}

Emerging data communication services create an increasing degree of uncertainty and dynamism in the traffic distribution across carrier networks. Examples are virtual private networks (VPNs) as well as remote storage and computing applications [1, 3]. For network design purposes, such services are best captured by the hose model [7, 10], which treats the node ingress or egress capacities as known constants but does not specify point-to-point demands. For example, the service level agreement (SLA) for a VPN customer who wants to interconnect several business sites using a carrier's network could just specify the peak rates at each ingress node but leave open the distribution of traffic to be sent between each node-node pair. It is then up to the carrier to efficiently route the traffic over the network.

Contemporary carrier networks are often built on circuit-switched core technologies, Internet protocol over synchronous optical network (IP-over-SONET), which offer high reliability and fast protection schemes. However, when traffic demands change over time, traditional circuit-switched network architectures lack bandwidth efficiency, which can lead to a severe underutilization of network resources. Moderate degrees of traffic dynamics, such as diurnal demand variations, can potentially be handled by advanced control plane concepts (e.g., the automatically switched optical network, ASON, based on generalized multiprotocol label switching, GMPLS [29]), but rapidly changing demand patterns cannot. In contrast, packet-switched backbone architectures, Internet protocol over wavelength division multiplexing (IP-over-WDM), provide the benefit of statistical multiplexing, and thus allow for a better utilization of network resources without the need for a dynamic control plane. However, there are significant drawbacks arising from purely packet-switched 
architectures. First, packet-switched networks examine and route the traffic at each node along a source-destination path. As we see below, this comes at a considerable cost, since packet router ports are substantially more expensive than the equivalent ports on a circuitswitched cross connect [24]. This fact continues to hold for multiprotocol label switching (MPLS) networks based on IP/MPLS routers, since MPLS label lookup is inherently more involved than SONET cross connecting. In addition, for larger networks, the need for routers to establish connectivity in the core can become a node scalability problem due to the difficulties in scaling packet routers [15]. Second, packet-based networks, by their very nature, use buffering at each node, which introduces packet loss and delay jitter, and makes quality-of-service (QoS) guarantees difficult to achieve. Third, packet-switched core networks do not meet the reliability and restoration constraints granted by circuit-switched networking infrastructure.

In an effort to combine the advantages of circuit-switched and packet-switched network architectures for rapidly changing demand patterns, Refs. [16, 20, 21, 27, 28] apply Valiant's scheme of randomized load balancing (RLB) [25] over a circuit-switched network. This previous work has focused on a variety of network performance measures including throughput, link congestion, and switch fan-out. The basic idea of RLB (see Subsection 4.B) is to route demands from network edge nodes in two phases. In the first (load balancing) phase, all nodes randomly distribute their traffic among all other nodes. In the second (routing) phase, each node processes the packets it received in phase 1 and sends them to their final destination. Since each packet is only processed once on its path from source to destination, the need for multihop packet routing using core routers is greatly reduced. In each of the two phases, traffic is carried on cost-effective circuit-switched (layer 1) core technology, without having to experience layer 2 or 3 processing at each node. The resulting RLB network offers SONET-grade reliability, and in many cases promises lower deployment cost than conventional architectures designed for dynamic traffic variations. Furthermore, delay jitter and QoS guarantees are likely to be met more easily than in packet-switched architectures, since all packets experience only a single stage of routing (and therefore only a single stage of buffering).

In this paper, we first address some inherent drawbacks associated with RLB. Most notably, we explore the inefficiency in distributing all traffic across the entire network. Based on these observations, we propose selective randomized load balancing (SRLB) [27], where RLB is performed across a limited number of carefully chosen hubs in the network. We point out the advantages of SRLB over the other architectures discussed in this paper. After computing an optimal set of hubs, we are able to give empirical evidence that SRLB is an attractive architecture combining the distinct advantages of RLB (resilience) and VPN-tree routing (network cost).

This paper is organized as follows. In Section 2 we give an overview of the considered network architectures, including some important definitions. In Section 3 we give some background on models for uncertain demands, robust optimization, and oblivious routing. In Section 4 we discuss two important examples of oblivious flow templates: shortest-path (SP) routing and VPN-tree routing. We show how previous work on VPNs can be applied to avoid excessive processing costs at the nodes. We observe that RLB can use significantly more link bandwidth than an optimal VPN-tree network, which motivates the introduction of SRLB in Subsection 4.C, forming the conceptual core of this paper. Section 5 is the main empirical section and provides detailed results based on the cost of the network elements needed to build the different architectures studied. We show by means of empirical examples how SRLB lowers network cost and improves delay jitter. In Section 6 we then discuss resource utilization for different network architectures and flow templates, and we show to what extent (depending on the flow templates) resource underutilization can become an advantage for IP/MPLS networks through statistical multiplexing in the presence 
of best-effort traffic. In this context, we also introduce the notion of the robustness premium to quantify the amount of overprovisioning that is needed to accommodate dynamic traffic demands. Finally, we conclude in Section 7.

\section{Network Architectures}

\section{A. Circuits}

A circuit is defined by two end nodes and some provisioned, dedicated capacity in a physical network. This capacity can be viewed as a point-to-point pipe that carries traffic unaffected between the specified end nodes of the circuit, with no packet processing, header lookup, or label lookup required along the way. In practice, a circuit may be implemented within the SONET hierarchy, or it may be a full wavelength channel in an optical network using optical add/drop multiplexers (OADMs). Note that an MPLS "circuit" is not a circuit within this definition, since MPLS requires packet processing in the form of label lookup at each node from source to destination. The role of MPLS is discussed in detail in Subsection 6.D.

\section{B. Packet (Hop) Routing and Circuit Provisioning}

All network traffic reaches its destination by following a sequence of circuits or hops; the particular choice of a sequence of hops is referred to as the hop routing. If traffic follows several hops, the intervening nodes, called routing nodes, must support the functionality to route traffic onto the next hop toward its destination. This may, for instance, be achieved by examining each Ethernet frame, IP packet header, or MPLS label in between two hops using Ethernet switches or IP/MPLS routers (interconnected by SONET or WDM circuits).

Circuit provisioning constraints specify how individual hops are implemented in the physical network. Most standard is that a circuit is identified with a single, capacitated path between its end points, but one may also consider circuits implemented as fractional flows; this is also called multipath routing and is implemented, e.g., using virtual concatenation (VCAT). In addition, the circuits used by hop paths may be provisioned statically or dynamically; in the latter case, the physical layer needs a control plane that dynamically adapts to changing traffic patterns (e.g., ASON and GMPLS).

\section{C. Network Architectures}

A network architecture refers to a collection of constraints on how traffic is routed at the packet layer. The architectures we consider are the following: single-hop routing, hub routing (dual-hop routing via one node), Valiant's randomized load balancing and selective randomized load balancing (dual-hop routing via all terminal nodes (RLB) or a selection of a subset of any nodes (SRLB)), and multihop routing (no bound on the hop length).

Given an architecture, there exist several physical implementations of the network depending, for instance, on the choice of switching (node) equipment.

\section{D. Examples}

We discuss several concrete examples of the abstract architectures considered. Figure 1(a) depicts a single-hop network architecture, where packets are routed at the ingress, where they are placed onto predefined circuits, and traverse a circuit-switched core network to their destinations. If static circuit provisioning is employed, each node-node pair $(i, j)$ has to be connected by a circuit of capacity equal to the maximum possible demand between $i, j$. Since every circuit can handle the entire demand originating at a node without reconfiguration, no control plane is needed, but the architecture results in a vast overprovisioning of network resources if traffic patterns are allowed to vary significantly. This can be mitigated by using dynamic provisioning of circuits by means of a dynamic control plane, 
setting up and tearing down circuits as needed, and thus allowing traffic to share core network resources. This approach is severely limited, however, by the speed and complexity of available control plane technologies.

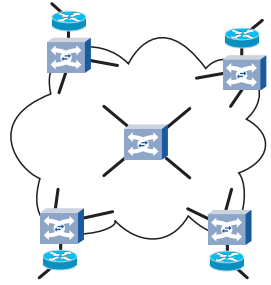

(a) Single-hop routing (e.g., 'IP-over-SONET')

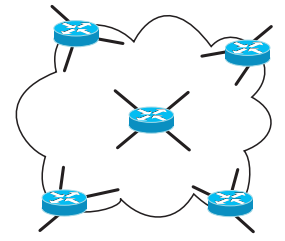

(b) Multi-hop routing (e.g., 'IP-over-WDM')

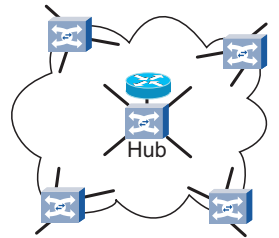

(c) Double-hop routing (Hub routing)

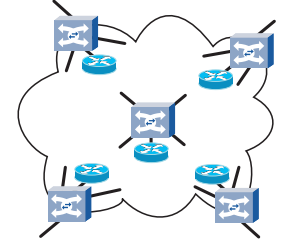

(d) Double-hop routing

Fig. 1. Network architectures considered in this work. (Square network elements: circuitswitched cross connects. Round network elements: packet routers.)

Figure 1(b) depicts a multihop architecture. Here, each core node examines the traffic entering it on a circuit and places it on a different circuit following a locally implemented routing strategy. The best-known example for this architecture is an IP/MPLS network, where nodes are IP/MPLS routers and circuits are point-to-point line systems (e.g., WDM links) between them. From a capacity analysis viewpoint, the multihop architecture is equivalent to a single-hop architecture with a sufficiently fast control plane to support traffic dynamism. The role of the globally acting control plane is taken over by statistical multiplexing through local packet routing.

Figures 1(c) and 1(d) depict the two extreme cases of double-hop architectures. Here, traffic is first sent to one (c) or more (d) intermediate routing nodes using preset circuits, irrespective of the traffic's final destination. The intermediate nodes perform local routing decisions, and again use the circuit-switched core to deliver traffic to its final destination. The case of a single intermediate routing node [Fig. 1(c)] is called a hub routing architecture. Although using a single hub often leads to lowest overall network cost, it is not the most desirable architecture in practice, since the hub (i) represents a single point of failure and (ii) has to route the entire network traffic, which can quickly become a network scalability problem. The case where incoming traffic is distributed across all nodes [Fig. 1(d)] is inspired by Valiant's randomized load balancing, introduced in the context of parallel computing [25] and discussed further in Subsection 4.B.

\section{Terminology and Background}

We consider network design where we are given a physical network represented by a graph $G=(V, E)$. The set $V$ represents node locations, and $E$ is the edge set, representing which nodes pairs are joined by a physical link. Our assumption is that each edge has an abundance of channels (for instance, fibers) and that network cost is mainly associated with (i) activating these channels (for instance, installing line terminal equipment or optical amplifiers along the line) and (ii) populating the nodes with the appropriate switching equipment (such as packet routers and SONET cross connects). Thus for our purposes, we view each edge $e \in E$ as having an unbounded supply of bidirectional (undirected) links, each with an associated cost for activating. The full cost model is discussed at length in Section 5.

We also have a specified set of edge nodes (sometimes called terminals), where traffic is injected into the network. Normally, we take the set of edge nodes to be the whole set $V$, but the algorithms work for any proper subset. Even though our edges are undirected (bidirectional), we maintain the convention that demands may be oriented; that is, there may be distinct unidirectional demands from node $i$ to node $j$, and from node $j$ to node $i$. 
In our numerical implementations, we normally think of these demands as being paired and then routed on bidirectional paths. Traffic is then specified by a demand matrix $d_{i j}$. We refer to the value $\sum_{j} d_{i j}$ (or $\sum_{j} d_{j i}$ ) as the ingress (or egress) traffic at $i$. We assume throughout that there is a bound denoted by $D_{i}$ (also called the marginal) on both the ingress and egress traffic at $i$.

\section{A. Uncertain Demands and Robustness}

Network designers have traditionally adopted the view that an accurate estimate for pointto-point traffic is given prior to laying out circuits. The increasing importance of flexible data services has led to situations where traffic patterns are either not well known a priori or are changing rapidly. In these settings the network should be dimensioned to support not just one traffic matrix but a large class of matrices determined by the application.

This scenario leads to a robust optimization problem [3, 4]: given a universe $U$ of socalled valid demand matrices, normally specified as a convex region, the goal is to design the network so that every demand matrix in $U$ can be supported at the lowest possible cost. The simplest form of this problem, recently shown to be nondeterministic polynomial time hard (NP-hard) [6], is to allocate fractional link capacities that are sufficient to support a multicommodity fractional routing for each demand matrix in the universe $U$, where the fractional routing used may change dynamically if the demand matrix does.

Motivated by the application of supporting uncertain or changing demands, the focus of our study is on the universe of hose matrices. In the hose model, there is a subset of nodes that inject traffic into the network. For each of these nodes $i$, we have a bound $D_{i}$ that is an upper bound on the total demand that this node may offer (ingress capacity) or receive (egress capacity). Note that in general, these bounds could be independent of each other The class of hose matrices for the marginal values $D_{i}$ is then $U=\left\{d_{i j} \geq 0: \sum_{j} d_{i j} \leq D_{i}, \sum_{j} d_{j i} \leq D_{i}, \forall i\right\}$. Recall that we do not in general have to assume symmetric demands, nor do we have to assume anything about demand from $i$ to $j$ using the same path as that from $j$ to $i$ (though in fact we could if we wish, without changing the results).

It is obvious that in general one must pay more to be able to support a whole class of demand patterns rather than just a single traffic matrix. We refer to this factor increase in cost as the robustness premium, and we report on its value from our experiments in Subsection 6.E.

\section{B. Oblivious Routing and Flow Templates}

The capacity required to support a class of traffic matrices obviously depends on the degree to which network elements can use current information about the network topology and utilization of network resources, for determining a cost-effective routing of the existing demands. Real-time reprovisioning of circuits in physical core networks to adapt to changing demands is not generally available. In contrast, packet switches (IP routers or Ethernet switches) have some ability to adapt traffic routes in real time, but this leads to a network management overhead since complex traffic engineering may be needed. For these reasons, in our study, we restrict ourselves to oblivious (sometimes called static [4]) routing strategies, which are agnostic of network utilization parameters or current traffic distributions.

Formally, an oblivious routing is determined by a flow template. A flow template for a specific node pair $(i, j)$ specifies how to send one unit of flow from $i$ to $j$. Hence it can be modeled as an assignment $f(P)$ to each $i-j$ path so that $\sum_{P \in P_{i j}} f(P)=1$. We use $P_{i j}$ to denote the set of paths (usually simple) between $i$ and $j$. If our network is currently handling a traffic matrix $d$, then the interpretation is that it treats the $i$ to $j$ traffic as follows: for each $i-j$ path $P$, it sends $f(P) d_{i j}$ flow along path $P$. Naturally, flow templates can 
be fractional (multipath routing) or single path (unsplittable flow), or they may obey some other restrictions, for instance, bounds on path length. A flow template, usually denoted by $f$, then consists of a template for each pair of nodes $i, j$ that are injecting traffic into the network. We have defined our templates in terms of a path variable; compact arc/edge formulations could have also been used as is done in Refs. [2, 9] for related problems. To avoid confusion, we note at this point that an oblivious routing strategy does not actually define a hop routing since it does not specify at which nodes packet routing is actually performed. Rather, flow templates specify the flow of traffic at the physical layer.

Optimization of demand-oblivious routings (for any network performance measure including cost) can be challenging since flow templates seek to perform resource sharing between different demand matrices. Work on finding efficient demand-oblivious routings for robust networks has focused on choosing the universe $U$ to be the matrices arising from the hose model [7-12, 14, 22]. We are especially interested in the work of Gupta et al. [11] on the uncapacitated, undirected version of the hose model with equal bounds on ingress and egress capacities. A different setting is considered in Ref. [23], where an efficient oblivious routing (with respect to link congestion as opposed to network cost) is described for the universe of demand matrices that are routable in the existing network (cf. also Ref. [6]).

We mention that the worst-case concept of robustness is only one way to approach uncertainty in optimization data. Others include chance-constrained optimization and stochastic programming. We refer the reader to Ref. [18] for an application of the latter approach to network design and subsequent augmentation.

\section{Examples of Flow Templates and Selective Randomized Load Balancing}

We consider several flow templates for oblivious routing: SP routing, tree routing, hub routing, and randomized load balancing. We see that in uncapacitated networks, RLB can be viewed as a "convex combination" of hub routing templates. This leads us to propose an intermediary selective randomized load balancing scheme.

\section{A. Virtual Private Network Trees and Hub Routing}

In this subsection, we discuss tree networks as highly attractive topologies to support hose traffic. As is obvious in Subsection 4.C, these discussions are crucial to transition from RLB to the newly proposed SRLB architecture.

Given any fixed tree $T$ that contains all edge nodes, the capacities required to support all traffic under the (undirected or bidirectional) hose model are readily computed as follows. For each edge $e \in T$, consider the two subtrees obtained after deleting $e$. Let $B$ be the smaller of the total marginal capacities (i.e., the sum of the node ingress and egress capacities) in each of the two subtrees. One easily sees that there is a valid hose matrix that simultaneously sends $B$ demand from the smaller subtree to the larger subtree and vice versa. Thus, if all hose matrices ought to be routed on $T, e$ must support a bidirectional capacity of at least $B$. We refer to the link-capacitated tree resulting from repeating this calculation for each edge $e$ as the VPN tree associated with $T$ and denote it by $\operatorname{VPN}(T)$; the name is inspired from the application to virtual private networks considered in Ref. [11]. The link capacities on any VPN tree are sufficient to route every hose traffic demand if we use the following oblivious flow template: demand between any node pair $i, j$ routes along the unique shortest path between $i$ and $j$ in $T$. We call this oblivious flow template the tree template associated with $T$. An elegant method for computing the optimal VPN tree is derived in Ref. [11].

We also consider a second (non-shortest-path) flow template on trees, called the hub routing template. For any tree $T$ and node $v$, consider the flow template where every incoming demand first sends traffic to the hub node $v$, and then $v$ forwards the demand to 
its final destination. Using this template, the link capacities required to support every hose matrix are easily computed. Namely, consider the load on each edge when the edge nodes $i$ simultaneously send $D_{i}$ bidirectional traffic to the hub node $v$. We denote the resulting linkcapacitated tree as $\operatorname{HUB}(T, v)$. It is easy to check that any edge in $\operatorname{HUB}(T, v)$ is assigned at least as much capacity as it is in $\operatorname{VPN}(T)$.

In their study of the VPN problem [11], Gupta et al. show a connection between the two types of capacitated trees $\operatorname{VPN}(T), \operatorname{HUB}(T, v)$. Namely, they show that there is an optimal capacitated tree (in terms of link capacity), denoted here by $T_{\mathrm{opt}}$, with the following simple structure. There is a node $r$ such that this tree is a shortest path tree $T_{r}$ rooted at $r$. If only some of the nodes are terminals, the tree is obtained by routing each edge node to $r$ along a shortest path to obtain a "cheapest flow tree" that contains all edge nodes. One consequence is that one may arrive at an optimal VPN tree simply by solving for a shortest path tree $T_{v}$ from each node $v$ and taking the cheapest of all resulting trees. A more important consequence is that in the optimal tree, their proof shows that $\operatorname{vpn}\left(T_{r}\right)$ is precisely the same as hub $\left(T_{r}, r\right)$. Thus there is enough capacity on the tree to use either the tree flow template as is done in Ref. [11] or the hub routing flow template. This is significant for us since we can implement a hub routing template via highly cost-efficient statically provisioned circuits between each node and the hub. In contrast to Ref. [11], where the focus is on link costs, this becomes significant for us since it eliminates the need to perform packet routing operations at nodes between source and destination, as would be required for tree routing. The difference between the two strategies is visualized in Fig. 2. In the remainder of the paper we use VPN tree and VPN hub to denote using the tree and hub flow templates, respectively, on an optimally designed tree $\operatorname{vpn}\left(T_{\mathrm{opt}}\right)$, defined above.

We close by mentioning that in Ref. [11] it is shown that the cost of vpn $\left(T_{\text {opt }}\right)$ is within a factor of 2 of the optimum possible by any fractionally capacitated network. It remains an open problem to determine whether it is in fact optimal! (cf. [13]).

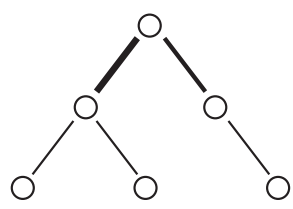

(a) Tree routing

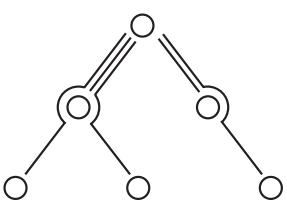

(b) Hub routing

Fig. 2. Tree routing (a) and hub routing (b) make use of different circuits (represented by lines), yet sometimes require identical edge capacities.

\section{B. Randomized Load Balancing}

Randomized load balancing is a two-step (double-hop) routing scheme based on a statically provisioned circuit-switched core. In a first (load balancing) phase, traffic originating at any node of an $N$-node network is uniformly distributed among all $N$ nodes. For example, in the case of equal node ingress or egress capacities, each node distributes $1 / N$ th of its traffic to each other node (and keeps $1 / N$ th to itself). The traffic distribution in phase 1 is random in the sense that it is totally agnostic of the demand matrix and does not require any routing decisions at the ingress. In phase 2 , each node performs local routing decisions on the traffic received in phase 1 and statistically multiplexes the traffic onto circuits leading to its final destination. Due to the random and uniform distribution of traffic in phase 1, the traffic distribution in phase 2 will also be uniform on average, with fluctuations being accommodated by buffering within the routing nodes.

We discuss some of the concrete advantages, issues, and solutions associated with implementing RLB in practice. 
Hardware Benefits: The link capacities required to perform the two phases of RLB are readily obtained as follows $[10,11,16]$. For traffic marginals $D_{i}$ at the nodes, the amount of traffic distributed in each phase is the product multicommodity flow [17] induced by the $D_{i}$, i.e., the traffic between nodes $i$ and $j$ is $D_{i} D_{j} / \sum_{l} D_{l}$. Note that the uniform nature of traffic in phases 1 and 2, regardless of the actual demand matrix to be routed, permits preallocation of static network circuits, which dramatically simplifies network design.

Since each node $j$ receives a total of $\sum_{i} D_{i} D_{j} / \sum_{l} D_{l}=D_{j}$ from all $N$ nodes (including itself), the node routing capacity required for phase 2 equals the total node ingress capacity. This corresponds to the minimum possible routing capacity as required for source-routed (single-hop) network architectures. However, full support of dynamically changing demand patterns is maintained through local routing, and no global control plane is needed.

Resequencing, Delay, and Jitter: Since RLB performs strict double-hop routing, all traffic is buffered only once (at the beginning of phase 2). This reduces random buffering delays when compared to a multihop network architecture, which buffers traffic at each node. One obvious disadvantage of RLB (as with any other architecture employing multipath flow templates) is the routing of traffic over paths with significant time-of-flight differences. The resulting delay spread potentially asks for packet reordering. Note, however, that these time-of-flight differences do not contribute to random delay jitter, but are fully predictable based on knowledge of the hop routing and flow template, and can thus be counteracted by deterministic delays at the ingress, intermediate, or egress nodes. Alternatively, traffic splitting at the ingress node can be performed on a per-flow basis, as explained below. The maximum propagation delay in RLB is about twice the time-of-flight delay of the longest path in the flow template, which restricts the geographic dimensions of such networks.

Resilience and Security: Due to the distribution of traffic among all routing nodes in phase 1 of RLB, the architecture is inherently vulnerable to routing node failures. However, RLB uses a multitude of routing nodes, as opposed to the hub architecture, in which the routing hub represents a single point of failure. Therefore, RLB can be made robust to routing node failure either by means of error-correcting coding or by means of backpressure protocols that throttle the ingress traffic by $1 / N$ should a routing node fail. Another interesting aspect of distributing the traffic across the network is the resulting resilience to eavesdropping attacks. In order to successfully intercept information, an adversary has to tap into almost all routing nodes. In conventional network architectures, tapping into a single routing node or into a single link can be sufficient to fully intercept information.

In closing, we want to mention that RLB can either be performed on layer 2 (Ethernet) or layer 3 (IP). Within each implementation of RLB, there are several traffic splitting strategies. For example, by properly controlling the traffic splitting at the ingress node, one can either make sure that flows are kept together and are thus always routed along the same paths, or that flows are always split up on a per-destination basis. While the first option entirely eliminates the resequencing problem, it may reduce the traffic uniformity established in the load balancing phase in the presence of exceedingly large flows. The second option ensures uniformity, but it requires a smart ingress capability that adds an expense to edge router equipment.

\section{C. Selective Randomized Load Balancing}

From a network capacity point of view, the optimum VPN-tree network VPN $\left(T_{\text {opt }}\right)$ (using either the tree flow template or the hub routing template) is always as good as (and usually better than) using RLB. To see this, notice that phase 1 traffic (as well as phase 2 traffic) in RLB can be written as a $1 / N$ convex combination of $N$ capacitated trees arising from routing $D_{i}$ flow from each $i$ to the root of a shortest path tree $T_{v}$, for each node $v \in V$ (or each edge node in general). Thus, the total capacity required by RLB is a $1 / N$ convex combination of the capacitated trees $\operatorname{HUB}\left(T_{v}, v\right)$. The cost of RLB is then at least the cost 
of $\operatorname{VPN}\left(T_{\text {opt }}\right)$ by the following argument. We have seen in Subsection 4.A, that the total capacity cost of $\operatorname{HUB}(T, v)$ (for any $T, v$ and hence also for $T_{v}, v$ ) is at least as large as that of $\operatorname{VPN}(T)$. Moreover, the total capacity cost of $\operatorname{VPN}(T)$ for any tree $T$ (and in particular for any $T_{v}$ ) is at least as large as the capacity cost of an optimal VPN tree $T_{\text {opt }}$. Thus the RLB capacity is a convex combination of trees, each of whose capacity cost is at least as large as that of $T_{\mathrm{opt}}$. Figure 3 visualizes this important observation.

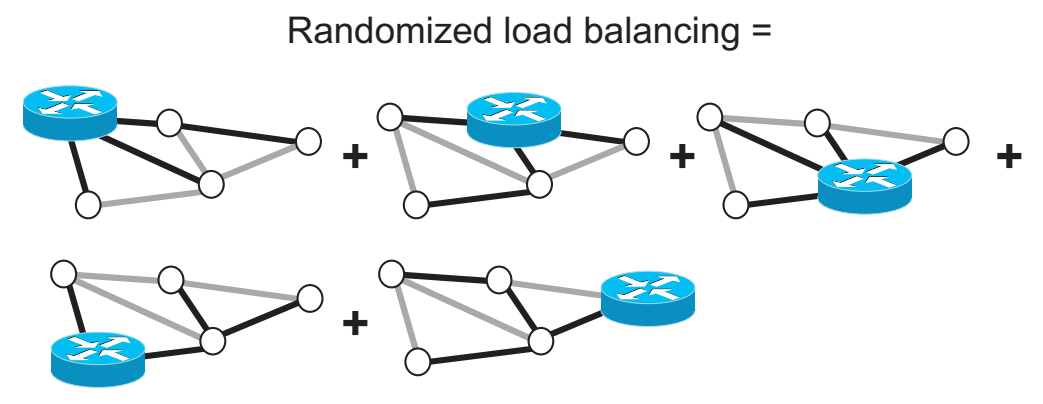

Fig. 3. RLB is a convex combination of hub routing templates. For each root node $v$, edges that are part of $T_{v}$ are shown in black.

As a consequence, we introduce SRLB as a blend of the two dual-hop architectures by performing RLB over those $M<N$ hubs that are associated with the $M$ best shortest path trees (computed over all nodes as hubs). The cost criterion for computing these $M$ best trees may be based on, e.g., network cost or on minimizing differential delay. For example, if the objective lies in minimizing network cost, one first calculates the cost of all $N$ shortest path trees (one for each node), and simply picks the $M$ lowest-cost trees. One then performs SRLB using the hubs from the selected trees.

With respect to reducing propagation delay and delay spread, we note that the hubs associated with the $M$ lowest-cost trees are typically clustered together near the center of the network; the "center" is characterized by the notion that the aggregate traffic on all edges connected to the center is best possibly balanced, reflecting directly the construction of the VPN tree [11]. Since lowest-cost nodes tend to be clustered together, the difference between the transport distances for any demand using these nodes as a routing hub is minimized. This mitigates one severe drawback of randomized load balancing: the different delays of packets distributed to different intermediate nodes (delay spread) and the potential resulting need for packet resequencing.

Another drawback revealed in our empirical studies in Section 5 is that when switching equipment costs are included, network cost based on RLB may actually exceed that of multihop IP designs. In contrast, SRLB across a limited number of hubs yields designs cheaper than either RLB or multihop architectures. A quantitative comparison of SRLB to RLB and to conventional network architectures in terms of cost, delay, and delay jitter is given in Section 5.

\section{Cost Comparison and Resource Requirements}

In this section, we compare the capacity requirements for the architectures and flow templates introduced in Sections 2, 3, using the three example networks depicted in Fig. 4: the UK research network JANET, the US research backbone Abilene, and the European research network GÉANT.

We assume symmetric demands $\left(d_{i j}=d_{j i}\right)$ and equal nodal ingress and egress traffic $\left(\sum_{i} d_{i j}=D / N\right.$ for each $\left.j\right)$. Demand patterns are allowed to vary under the hose constraint, and all architectures are capacitated to accommodate all valid demand matrices. 


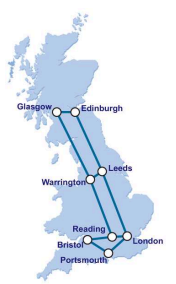

(a) JANET topology

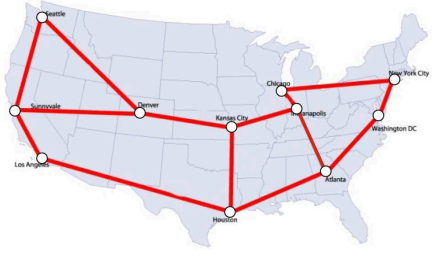

(b) ABILENE topology

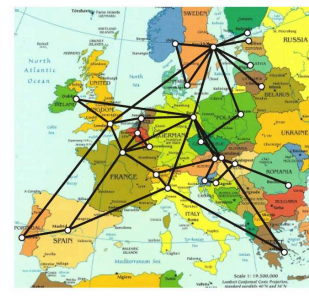

(c) GEANT topology

Fig. 4. Three example networks considered in this paper (http://www.ja.net, http://www.abilene.iu.edu, and http://www.geant.net).

As described in Appendix B, we use linear programming (LP) formulations to calculate the capacity requirements needed to support all hose demand matrices. Given a fixed flow template, one computes for each link or node the worst case capacity requirement needed to support every hose matrix. Each of these subproblems (one for each link or node) amounts to a so-called fractional $b$-matching problem $[2,9]$.

The results are displayed in Tables $1,2,3$. The first columns specify the network architectures, and the second columns indicate the flow template used. The tables list the total required circuit-switching capacity (e.g., by SONET cross connects), the total packetswitching capacity (e.g., by IP/MPLS routers), and the transport capacities (e.g., by WDM line systems) for different network architectures and shortest path (SP) as well as VPN-tree templates. The rightmost columns give the overall network cost, normalized to the hub architecture using an optimum VPN tree. In order to arrive at the overall network cost, we assume the following cost model for commercially available networking hardware [21, 24],

$$
c_{\mathrm{IP}-\text { port }}: c_{\text {SONET-port }}: c_{\mathrm{WDM} / \mathrm{km}}=370: 130: 1,
$$

where $c_{\mathrm{IP} \text {-port }}$ is the cost of an IP/MPLS router port, $c_{\text {SONET-port }}$ is the cost of a SONET cross connect port, and $c_{\mathrm{WDM} / \mathrm{km}}$ is the cost of WDM transport per kilometer of link distance, all for the same data rate. Note that our cost model breaks down cost directly to ports on routers or cross connects. In practice, this is a justifiable simplification, since the cost of line cards typically dominates the cost of main frames. Since we are giving all capacity numbers as well as cost numbers, it is possible for the interested reader to plug any other suitable cost ratio into our results.

For the RLB architecture, we assume that those line cards on the SONET cross connects handling the nodal ingress and egress traffic are equipped with means of packet (or flow) splitting and, if required, resequencing. We allocate an additional cost of half the cost of a standard circuit-switched line card to this functionality; thus, the per-port cost of an ingress and egress line card in the load-balanced architecture amounts to $1.5 c_{\text {SONET-port }}$.

For the circuit-switched network with dynamic control plane, we do not allocate any additional cost, since highly dynamic control planes do not yet exist, and a meaningful quantification of their cost cannot be given.

As is evident from the overview tables, the static single-hop architecture with its need for high overprovisioning leads to overly expensive network cost. Neglecting the dynamic control plane architecture for its lack of availability, the most important contenders for dynamic networking are the multihop (architecture 3), the RLB (architecture 4), and the hub (architecture 5). Of these three architectures, the hub architecture (using the optimum network node as a routing hub) proves cheapest of all networks, in agreement with the VPN-tree strategy $[11,22]$. However, all traffic is processed in a single routing node that has to be able to handle the entire network traffic $D$. Therefore, this architecture incorporates a single point of failure and is thus often considered unreliable. 
Table 1. JANET-Overview

\begin{tabular}{ll||cccc}
\hline Architecture & $\begin{array}{l}\text { Flow } \\
\text { Template }\end{array}$ & $\begin{array}{c}\text { Circuit-switching } \\
\text { capacity }\end{array}$ & $\begin{array}{c}\text { Packet-switching } \\
\text { capacity }\end{array}$ & $\begin{array}{c}\text { Transport } \\
\text { capacity } \times \mathrm{km}\end{array}$ & $\begin{array}{c}\text { Cost ratio } \\
\text { (to hub) }\end{array}$ \\
\hline 1. Single-hop & SP & 120 & 16 & 11,104 & 3.12 \\
\hline 2. Single-hop & SP & 42 & 16 & 3,437 & 1.42 \\
(dynamic) & VPN-tree & 32 & 16 & 2,302 & 1.18 \\
\hline 3. Multi-hop & SP & - & 42 & 3,437 & 1.81 \\
& VPN-tree & - & 32 & 2,302 & $\mathbf{1 . 3 5}$ \\
\hline 4. Load-balanced & SP & 44 & 8 & 2,776 & $\mathbf{1 . 1 4}$ \\
\hline 5. Hub routing & VPN-hub & 40 & 8 & 2,302 & 1.00 \\
\hline
\end{tabular}

Table 2. Abilene-Overview

\begin{tabular}{ll||cccc}
\hline Architecture & $\begin{array}{l}\text { Flow } \\
\text { Template }\end{array}$ & $\begin{array}{c}\text { Circuit-switching } \\
\text { capacity }\end{array}$ & $\begin{array}{c}\text { Packet-switching } \\
\text { capacity }\end{array}$ & $\begin{array}{c}\text { Transport } \\
\text { capacity } \times \mathrm{km}\end{array}$ & $\begin{array}{c}\text { Cost ratio } \\
\text { (to hub) }\end{array}$ \\
\hline 1. Single-hop & SP & 287 & 22 & 165,478 & 6.07 \\
\hline 2. Single-hop & SP & 71 & 22 & 37,019 & 1.57 \\
(dynamic) & VPN-tree & 51 & 22 & 22,621 & 1.08 \\
\hline 3. Multi-hop & SP & - & 71 & 37,019 & 1.82 \\
& VPN-tree & - & 51 & 22,621 & $\mathbf{1 . 1 9}$ \\
\hline 4. Load-balanced & SP & 72 & 11 & 30,087 & $\mathbf{1 . 2 7}$ \\
\hline 5. Hub routing & VPN-hub & 62 & 11 & 22,621 & 1.00 \\
\hline
\end{tabular}

Table 3. GÉANT-Overview

\begin{tabular}{ll||cccc}
\hline Architecture & $\begin{array}{l}\text { Flow } \\
\text { Template }\end{array}$ & $\begin{array}{c}\text { Circuit-switching } \\
\text { capacity }\end{array}$ & $\begin{array}{c}\text { Packet-switching } \\
\text { capacity }\end{array}$ & $\begin{array}{c}\text { Transport } \\
\text { capacity } \times \mathrm{km}\end{array}$ & $\begin{array}{c}\text { Cost ratio } \\
\text { (to hub) }\end{array}$ \\
\hline 1. Single-hop & SP & 2,157 & 54 & 760,210 & 15.87 \\
\hline 2. Single-hop & SP & 223 & 54 & 69,142 & 1.77 \\
(dynamic) & VPN-tree & 127 & 54 & 36,823 & 1.10 \\
\hline 3. Multi-hop & SP & - & 223 & 69,142 & 2.27 \\
& VPN-tree & - & 127 & 36,823 & $\mathbf{1 . 2 5}$ \\
\hline 4. Load-balanced & SP & 212 & 27 & 56,312 & $\mathbf{1 . 4 3}$ \\
\hline 5. Hub routing & VPN-hub & 154 & 27 & 36,823 & 1.00 \\
\hline
\end{tabular}

Depending on the network size, we identify RLB across all network nodes to be lowest cost on networks of smaller geographic size (JANET), while multihop VPN-tree routing performs better on larger networks (Abilene and GÉANT). This is expected from our previous discussions, since RLB in general uses up more transport capacity than VPN-treebased architectures, and can therefore only be proved if the cost of routing dominates the cost of transport. In fact, if we scale the Abilene topology from its average link distance of $1,317 \mathrm{~km}$ down to $831 \mathrm{~km}$, and the GÉANT topology from an average link distance of $797 \mathrm{~km}$ down to $319 \mathrm{~km}$, randomized load balancing exhibits equal cost to multihop routing on a VPN tree. For comparison, JANET has an average link distance of $184 \mathrm{~km}$, and randomized load balancing outperforms multihop IP routing up to an average link distance of $1,030 \mathrm{~km}$ on this topology. Note that conventional multihop routing on shortest paths is always more expensive than RLB on the studied networks.

\section{A. Selective Randomized Load Balancing}

We now examine SRLB across an optimally chosen subset of $M$ hubs. The process for choosing the hubs is as follows (see also Subsection 4.B): We evaluate the cost of each of the $N$ possible single-hub architectures using shortest path trees; the cheapest of these $N$ 
hub architectures, routing on an optimal VPN tree $[11,22]$, is listed under architecture 5 in Tables 1, 2, 3. The most expensive hub architecture exceeds the cost of the cheapest hub by $22 \%, 57 \%$, and $90 \%$ for JANET, Abilene, and GÉANT, respectively. We then sort our $N$ possible hub architectures in ascending cost and pick the $M$ lowest-cost hubs to perform SRLB.

Figure 5 quantifies the benefit of SRLB by showing the cost of a SRLB network, normalized to hub routing, as a function of the $M$ lowest-cost intermediate nodes, taking into account the cost of the traffic-splitting and resequencing hardware. Comparing the curves with the cost numbers for multihop VPN-tree routing from Tables 1, 2, 3, indicated by horizontal arrows to the right of Fig. 5, we see that SRLB on the Abilene network performs better than multihop VPN-tree routing if $M \leq 8$ intermediate nodes are chosen. On the GÉANT network, $M \leq 13$ intermediate nodes need to be chosen in order to compete against multihop VPN-tree routing. On the JANET network, RLB performs better than VPN-tree routing to start with, and SRLB is able to further reduce network cost.

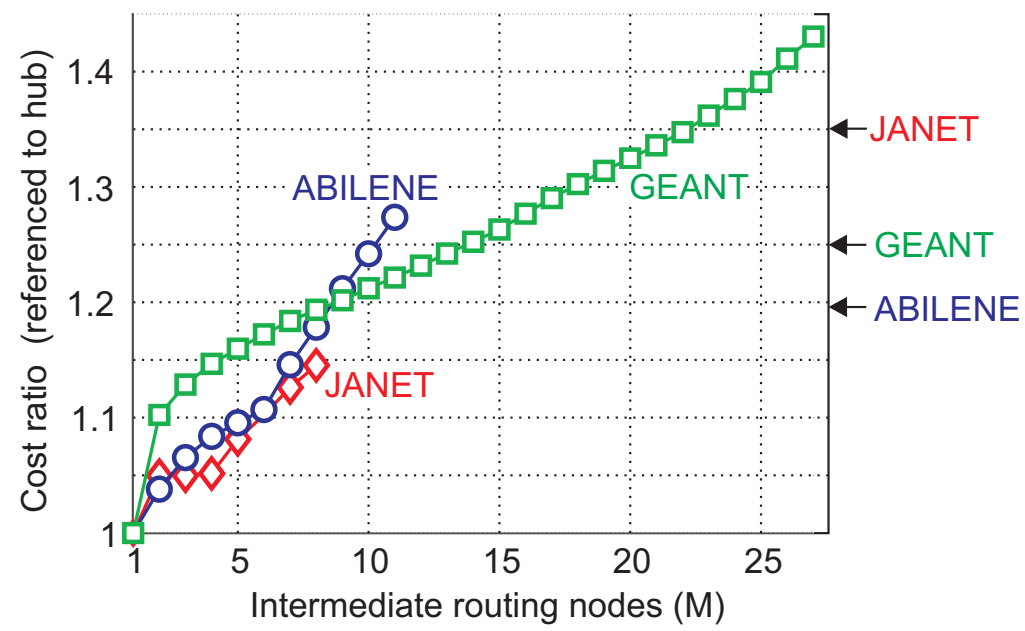

Fig. 5. Cost of selective randomized load balancing compared to cost of optimum hub routing as a function of number of intermediate nodes.

As explained in Subsection 4.C, SRLB mitigates the delay spread associated with the RLB architecture, since the lowest-cost hubs are located around the center of the network. To quantify this statement, we calculated the delay spread for RLB and SRLB on our three example networks. To this end, we evaluated, for every source-destination node pair $i, j$, the time-of-flight differences incurred by routing via all possible intermediate routing hubs. Our analyses show that the worst-case delay spread on the JANET network is cut in half by SRLB with 5 intermediate nodes instead of load balancing across the entire network. On the Abilene network, the same reduction is obtained when using 6 routing nodes, and on the GÉANT network when using 16 nodes.

\section{Resource Utilization, Classes of Service, and Robust Design}

In order to arrive at more detailed results for network capacity requirements, classes of service, and network resource utilization, we followed two independent avenues. First, we performed extensive Monte Carlo simulations using ensembles of randomly chosen demand matrices satisfying the hose constraint. Second, we solved the inherent $L P$ formulations for the problems. This allows for fast prediction of upper capacity bounds for each link and node. We combined the two approaches to measure resource utilization and the advantages 
of IP/MPLS due to statistical multiplexing.

\section{A. Statistics through Monte Carlo Simulations}

We performed Monte Carlo simulations using ensembles of 100,000 randomly chosen symmetric demand matrices satisfying the hose constraint, i.e., $d_{i j}=d_{j i}, d_{i i}=0$, and $\sum_{i} d_{i j}=\sum_{j} d_{i j}=D \forall i, j$. All $d_{i j}$ were allowed to vary between 0 and $D$ as long as the above constraints were met. The method of generating the random matrices is described in Appendix A. We routed each matrix individually across the network, using a fixed flow template (SP). We studied both minimum-hop and minimum-distance routing, with little quantitative difference for our three example networks. The results presented under "shortest path" in this paper refer to minimum-distance routing. For each matrix, we recorded the capacity needed on each link as well as at each node, leading to ensembles of 100,000 random link and node capacities, on which we performed statistical analyses. Figure 6 shows some selected results for the JANET network [Fig. 4(a)] using shortest path routing; (a) and (b) show histograms of the traffic flowing over two selected links (Glasgow-Edinburgh and Leeds-London), while (c) and (d) show histograms of the total traffic (add or drop plus through) to be handled by two selected nodes (Warrington and Leeds). The solid curves correspond to the tight hose model $\left(\sum_{i} d_{i j}=D\right)$, while the dotted curves apply to the weak hose model $\left(0 \leq \sum_{i} d_{i j} \leq D\right)$, where the total node demands were allowed to vary randomly between 0 and $D$ with uniform probability. The latter case models burstiness not only in the traffic distribution but also in the total node traffic demand. Note that the histograms may differ significantly from Gaussians, which are sometimes assumed in the context of evaluating packet loss or blocking probabilities.
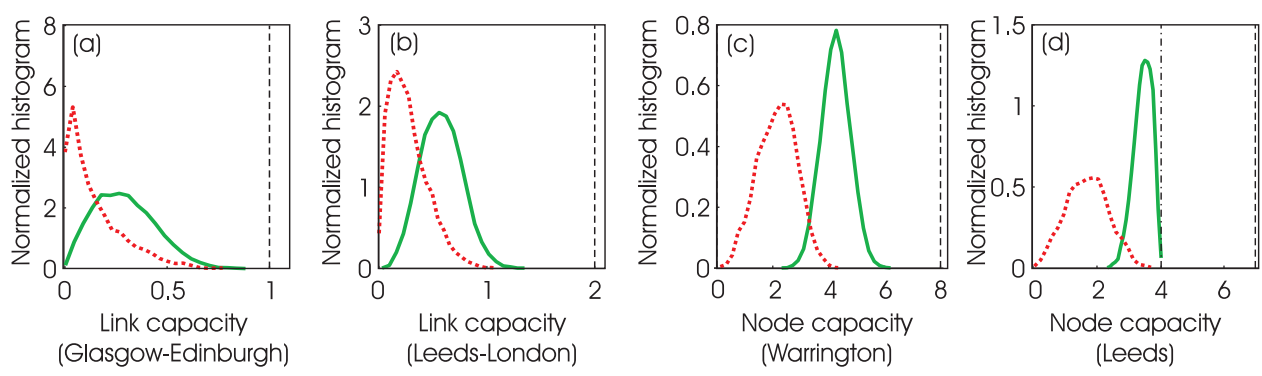

Fig. 6. Statistical analysis of the JANET network (100,000 realizations of a hoseconstrained, random demand matrix). (a) and (b) Histograms of the traffic on the links Glasgow-Edinburgh and Leeds-London. (c) and (d) Histograms of the total traffic (add/drop and through) handled by nodes Warrington and Leeds. Solid curves: Tight hose model $\left(\sum_{i} d_{i j}=D\right)$. Dotted curves: Weak hose model $\left(0 \leq \sum_{i} d_{i j} \leq D\right)$ with uniformly distributed node traffic. Dashed lines: Worst-case link capacities in (a) and (b); worst-case node I/O capacities in (c) and (d). Dash-dotted line in (d): Worst-case node switching capacity.

\section{B. Upper Bounds through Linear Programming Formulations}

The dashed curves in Figs. 6(a) and 6(b) show the maximum (worst-case) capacities that have to be expected over the two links, obtained by solving the corresponding LP formulations described in Appendix B. These lines represent cutoffs in the displayed histograms. A close analysis of the histograms showed that the upper bounds found by the LP formulations are indeed approached in our ensemble of 100,000 realizations, indicating good coverage of the demand distribution space by our random matrix algorithm. 
The input-output $(I / O)$ port capacity at a node is equal to the sum of the maximum capacities on links incident to that node. These maximum node I/O capacities are represented by the dashed curves in Figs. 6(c) and 6(d). Note that the histograms in Fig. 6(d) are not tightly bounded by the maximum I/O capacity. This is because the maximum traffic being switched at a node can generally be smaller than the maximum I/O capacity. In other words, there is no specific reason why there should be some valid demand matrix that simultaneously maximizes the load on each link into some node. This is exhibited by the dash-dotted curve in Fig. 6(d), which shows the required node switching capacity, computed by means of a node-centered LP problem discussed in Appendix B. Although one may hope to seek a cost advantage when the node switching capacity requirement is less than the node I/O capacity requirement, routers and cross connects are typically designed so that their switching capacity equals their I/O capacity; thus, it is the worst-case I/O capacity rather than the worst-case node switching capacity that drives node design. In the rest of this paper, we therefore always use I/O capacities when speaking about node capacity requirements.

\section{C. Resource Utilization}

Figure 7 shows histograms obtained for the capacities on two links (Warrington-Leeds and Warrington-Reading) of the JANET network, assuming the tight hose constraint $\left(\sum_{i} d_{i j}=D\right)$. The solid curves apply to SP routing, and the dashed curves represent tree routing on the optimum VPN tree $T_{\text {opt }}$ (VPN tree), which in this case is rooted at Warrington. As in Figs. 6(a) 6(b), the dashed vertical lines represent the upper capacity bounds obtained by the associated LP formulations; for the two links displayed, the upper bounds happen to be the same for both flow templates. However, the shift of the histograms toward higher capacity values for VPN-tree routing indicates better resource utilization through dynamic traffic aggregation [11,22].
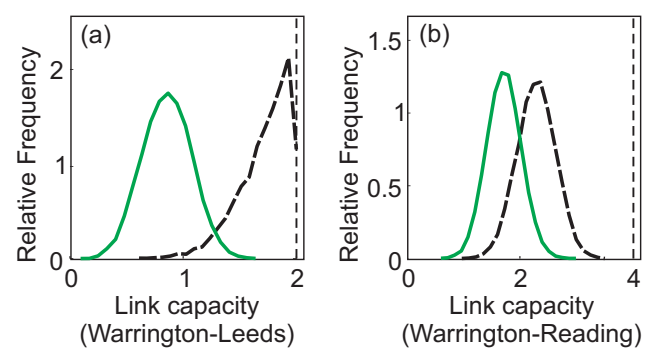

Fig. 7. Histograms for two links on the JANET network using SP routing (solid) and VPNtree routing (dashed). Dashed vertical lines indicate upper capacity bounds found by LP formulations.

Table 4 summarizes the average network resource utilization for the three example networks of Fig. 4 using SP as well as VPN-tree routing. The first row lists the number of links used by the two flow templates. The numbers $\left\{u_{l}, u_{n}\right\}$ represent the network-averaged utilization of link capacities $\left(u_{l}\right)$ and node capacities $\left(u_{n}\right)$, defined as the percentage fraction of the mean capacities flowing over a link or through a node to the capacities that have to be provisioned to satisfy full traffic dynamism under the hose constraint. The numbers clearly reflect the better network utilization achieved by VPN-tree routing as compared to SP routing. In addition, the number of links used by the VPN-tree template is smaller then used by the SP template. 
Table 4. Average Capacity Utilization

\begin{tabular}{l|cccccc}
\hline & \multicolumn{2}{|c}{ JANET } & \multicolumn{2}{c}{ ABILENE } & \multicolumn{2}{c}{ GÉANT } \\
& SP & VPN-tree & SP & VPN-tree & SP & VPN-tree \\
\hline $\begin{array}{l}\text { links } \\
\begin{array}{l}\text { average capacity } \\
\text { utilization [\%] }\end{array}\end{array}$ & 10 & 7 & 14 & 10 & 40 & 26 \\
\hline
\end{tabular}

\section{D. Multiprotocol Label Switching and Classes of Service}

So far, we have considered only a single class of traffic and have demanded that the studied network architectures together with their associated flow templates should be able to accommodate the hose constraint for all ingress traffic. We now extend our analyses to the case of two traffic classes. We investigate the benefits of statistical multiplexing in IP/MPLS networks and study how this benefit relates to RLB and SRLB architectures.

One of the main advantages of MPLS, and packet routing in general, is its ability to take advantage of statistical multiplexing to accommodate best-effort traffic. Thus, labelswitched paths in MPLS represent "soft circuits" in the sense that capacity currently unused for high priority (class A) traffic may be used for lower priority (class B) traffic. This way, the drawback of resource underutilization, discussed in Subsection 6.C, is turned into an advantageous feature. To quantify the benefits of classes of service in IP/MPLS networks, we design an IP/MPLS network that guarantees a certain amount of class A traffic under the hose constraint. We then calculate the average amount of class B traffic that can be carried on top of this class A traffic by filling idle resources. These results are contrasted with the RLB network design, which by its very nature always utilizes all network resources to their full extent and always provides guaranteed (class A) connectivity for the entire traffic.

Assuming the tight hose constraint $\left(\sum_{i} d_{i j}=D\right)$ for all three networks of Fig. 4, we partition the hose traffic $D$ into class A hose traffic $D_{A}=\alpha D$ and class B hose traffic $D_{B}=$ $(1-\alpha) D$. For each value of $\alpha$, we dimension the network to guarantee all possible class A demand matrices using the LP formulations described in Appendix B. We then randomly generate ensembles of 1,000 class A matrices and route them on our networks. We record the link and node capacities used to route all class A demands. For each of the 1,000 class A matrices, we then generate 100 random class B matrices and greedily route as many class B demands as possible in random order, given the available capacities that are not used for the particular realization of class A traffic. We thus generate an ensemble of 100,000 random realizations of class B traffic that is permitted by the network and take the average as our figure of merit. Figure 8 shows the average class B traffic supported by the network as a function of the amount of class A traffic $\alpha$, both expressed as a percentage of the total hose traffic $D$. Solid curves apply to SP routing, while dashed curves represent VPN-tree routing. As expected from our discussions on resource utilization, SP routing allows for more class B traffic than VPN-tree routing, since it utilizes network resources to a smaller extent. Using VPN-tree routing, at most a few percent of the hose traffic can be routed as class B traffic, which demonstrates that the benefit of "soft" circuits in combination with statistical multiplexing largely disappears for VPN-tree routing. For SP routing, the studied IP/MPLS networks support up to $18 \%$ of the hose traffic as class B traffic, but they also reject a significant amount of class B traffic. For example, designing the JANET network using SP routing to support $50 \%$ of the node ingress traffic as class A traffic, the network allows for an additional $14 \%$ of the ingress traffic being sent as class B traffic, while $36 \%$ of the best-effort ingress traffic has to be dropped.

To design an RLB network that supports the same average traffic as the IP/MPLS net- 


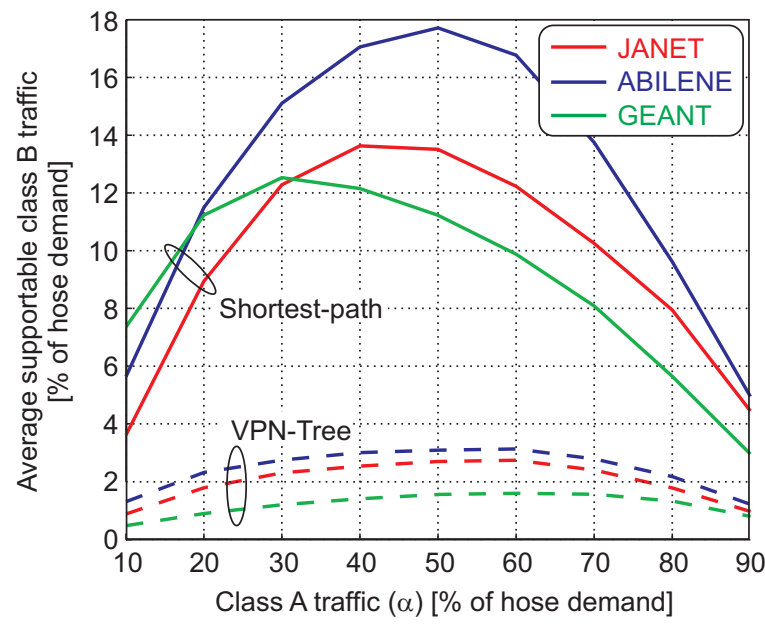

Fig. 8. Average class B traffic supported by the network as a function of class A traffic $\alpha$, both expressed as a percentage of the total hose traffic $D$. Solid curves apply to SP routing, dashed curves represent VPN-tree routing. The underlying networks are shown in Fig. 4.

work, we take the amount of class A traffic plus the average amount of class B traffic carried by the IP/MPLS network as the total ingress traffic to the RLB network. In the above numeric example for JANET, the resulting RLB network therefore needs to support $50 \%+14 \%=64 \%$ of the hose traffic. This implies that in order to be cost competitive, $64 \%$ of the cost of an RLB network needs to be lower than $50 \%$ of the cost of a multihop IP/MPLS network using SP routing. Recalling the results from our cost analysis in Tables 1, 2, 3, this indeed holds true for JANET: $64 \%$ of the cost of RLB on JANET is still $20 \%$ less expensive than multihop IP/MPLS using SP routing.

\section{E. Robustness Premium}

In this subsection we examine the following question: How much more capacity is needed to support all hose-constrained matrices (or any fixed universe of demand matrices) compared to just supporting a single benchmark demand matrix among them? We refer to the ratio of these link capacity costs as the robustness premium. The robustness premium obviously depends on the universe of demand matrices as well as on the choice of the benchmark matrix. Given that we are working with the class of hose matrices, it is natural to use as our benchmark matrix the product multicommodity flow matrix $u$, where $u_{i j}=D_{i} D_{j} / D \forall i, j$ and $D=\sum_{l} D_{l}$. (If all marginals are equal, this simplifies to the uniform multicommodity flow.)

The robustness premium naturally depends on the choice of a flow template since it determines how hops are transported in the physical layer. General robust network design problems actually treat the flow template as a variable in the optimization problem. Our study, however, focuses on a limited number of network architectures, which we can evaluate individually. In this subsection we present results on the robustness premium in terms of link costs only. Thus we effectively consider only three templates. The first is where every node pair routes their demand along a single shortest path. The second is where we are given the optimal VPN tree, and node pairs send flow on a simple path in the tree. We actually have two possible flow templates for trees (as discussed in Subsection 4.A; see Fig. 2) but they are equivalent in terms of link cost. The last template considered is RLB.

Table 5 gives our empirical results for the robustness premium on the three specific networks. We used the link capacity information developed in Section 5 and listed in Tables 
$1,2,3$. We have thus assumed that all ingress capacities are equal, and so we use the uniform demand matrix as our benchmark. Since we are dealing with uncapacitated networks, the cost of using RLB is simply twice that of satisfying this uniform demand. Note that the premium for using $S P$ routing is substantially more on all networks. Routing on an optimal VPN tree $T_{\text {opt }}$ significantly reduces the required resources, consistent with Refs. [11, 22]. Moreover, the premium for using VPN trees decreases as network size increases.

Note that the robustness premium in Table 5 is based on link costs only. Accounting for node costs (according to Tables 1,2,3) reveals that RLB, and in particular SRLB, is more advantageous than multihop routing using the VPN tree.

Table 5. Robustness Premium

\begin{tabular}{cccc}
\hline Flow Template & JANET & Abilene & GÉANT \\
\hline 1. SP & 2.48 & 2.46 & 2.46 \\
2. VPN-Tree & 1.66 & 1.50 & 1.31 \\
3. RLB & 2 & 2 & 2 \\
\hline
\end{tabular}

\section{Conclusions}

We have seen that optimal VPN trees for hose matrices in uncapacitated networks can be used to support hub routing instead of direct routing. Since randomized load balancing (RLB) can be viewed as a convex combination of hub routing from different hubs, we propose selective randomized load balancing (SRLB) to achieve costs similar to optimal VPN trees, yet maintaining the benefits of RLB. We benchmarked SRLB as well as RLB against other single-hop, dual-hop, and multihop circuit-switched and packet-switched network architectures using SP and VPN-tree routing. Using three representative carrier networks as examples, we investigated the cost of these architectures to support dynamically changing demand patterns. Our analyses take into account the cost relationship between switching, routing, and transport equipment. Further work should incorporate more restrictions on the universe of valid matrices, such as in Refs. [1, 2, 20]. Work on the benefits of hierarchical (geographical) hubbing is also needed, as is a more thorough treatment of issues related to network resilience and restoration. Finally, it will be interesting to understand better the algorithmic issues in capacitated networks; this has already been addressed in Ref. [16] with respect to throughput.

\section{A. Appendix A}

In this appendix, we describe how we generated a set of randomly chosen demand matrices satisfying the hose constraint.

We sample the set $D$ of all possible symmetric $N \times N$ demand matrices $\left[d_{i j}\right]$ associated with given capacities of the network nodes $D_{i} \geq 0, i=1, \ldots, N, N \geq 4$, more or less uniformly using a standard $\mathrm{M}($ arkov $) \mathrm{C}($ hain $) \mathrm{M}$ (onte) $\mathrm{C}$ (arlo) algorithm. Recall that $d \in D$ is characterized by the conditions

$$
d^{T}=d \geq 0, \quad d_{i i}=0, \quad \sum_{j=1}^{n} d_{i j}=D_{i}, \quad i=1, \ldots, N .
$$

Our MCMC algorithm starts with an arbitrary seed from $D$ and applies the following transition rule over and over: If $d \in D(\mathbf{c})$ is the current demand matrix, then we first choose randomly an index quadruple $1 \leq i<j<k<l \leq N$, and we determine the maximal interval $t \in\left[t_{0}, t_{1}\right]$ such that

$$
d(t):=d+t\left(E_{i j}-E_{j k}+E_{k l}-E_{l i}\right) \in D .
$$


Here the matrices $E_{i j}(i \neq j)$ have exactly two nonzero elements (at positions $i j$ and $j i$ ), and their union forms a basis in the set of all symmetric matrices with zero diagonal. Since $E_{i j}-E_{j k}+E_{k l}-E_{l i}$ has always zero row sums, all updates given by Eq. (A2) automatically satisfy the capacity constraints, and $t_{0}, t_{1}$ can easily be found from the remaining constraint $d(t) \geq 0$ :

$$
t_{0}=-\min \left(d_{i j}, d_{k l}\right), t_{1}=\min \left(d_{j k}, d_{l i}\right) .
$$

Then, choosing $t$ randomly from the interval $\left[t_{0}, t_{1}\right]$, we take $d(t)$ as the next demand matrix. Note that the transition rule requires a constant amount of computations, including calls to a standard random number generator.

It can be shown that, for any initial seed $d_{0} \in D$ the ensembles $\left\{d_{0}, d_{1}, \ldots, d_{n}\right\}$ obtained by successively applying the transition rule converge to a uniform distribution on $D$, as $n \rightarrow \infty$. Since experimentally good mixing was achieved for the ensemble sizes used in the simulations, we did not investigate the mixing time (generally, as $N$ grows larger, $n$ is required to achieve close to uniform sampling). Initial seeds can easily be determined for any set of feasible capacities by induction in $N$ (the feasibility condition says that $\left.2 \max D_{i} \leq \sum D_{i}\right)$. For $N=2,3$ and feasible capacities, or for $N \geq 4$ and $2 \max D_{i}=\sum D_{i}$, the set $D$ consists of one element.

\section{B. Appendix B}

We briefly outline the linear programs used to compute the link and nodal capacities required to support all demands under the hose model if we have some fixed single-path flow template $f$. For link capacities this is just the undirected version of LPs given in Refs. [2, 9], and so we focus on the LP for computing the maximum node switching capacity required. The following must be solved for each node $v$ in the network. Let $P_{v}$ denote those paths $P$ containing $v$ as an internal node, and let $f(P)=1$. Note that even if $f$ is a SP routing, there may still be shortest paths containing $v$ but that are not contained in $P_{v}$. We create an auxiliary directed graph $G^{\prime}=\left(V, E^{\prime}\right)$ as follows. The node set of $G^{\prime}$ is the set of edge nodes (we have nominally been taking this to be all nodes) in the original physical network. If there is some $P \in P_{v}$ from node $i$ to $j$, then we add an edge $(i, j)$ to $E^{\prime}$. The capacity of the switching fabric at $v$ required to support all hose demand matrices is equivalent to finding a maximum $d$-matching in $G^{\prime}$, where $d=\left(D_{1}, D_{2}, \ldots, D_{n}\right)$. In other words, we seek a solution to the following LP. Maximize $\sum_{(i, j) \in E^{\prime}} x_{i j}$ subject to $x \geq 0$ and for each node $i \neq v, \sum_{j} x_{i j} \leq D_{i}$, and $\sum_{j} x_{j i} \leq D_{i}$. Note that edge $(i, j)$ is distinct from $(j, i)$ only because we chose to adopt the practice of treating traffic from $i$ to $j$ separately from traffic from $j$ to $i$. Though in implementation we have always computed equal and oppositely directed demands between any pair (that in addition use the same paths in opposite directions). Similarly, for link capacities we form the same LP, but instead it is based on using $P_{e}$, the set of paths $P$ containing $e$ and with $f(P)=1$.

\section{Acknowledgments}

We are grateful to Peter Oswald who implemented the procedure for randomly generating demand matrices (Appendix A). We also thank Martin Zirngibl for valuable discussions.

\section{References and Links}

[1] A. Altin, E. Amaldi, B. Pelotti, and M. Pinar, "Provisioning virtual private networks under traffic uncertainty," presented at the International Network Optimization Conference, Lisbon, Portugal, March 2005.

[2] D. Applegate and E. Cohen, "Making intradomain routing robust to changing and uncertain traffic demands: understanding fundamental tradeoffs," in Proceedings of the ACM SIGCOMM 
2003 Conference on Applications, Technologies, Architectures and Protocols for Computer Communications (Association for Computing Machinery, 2003), pp. 313-324.

[3] W. Ben-Ameur and H. Kerivin, "New economical virtual private networks," Commun. ACM 44(6), 69-73 (2003).

[4] W. Ben-Ameur and H. Kerivin, "Routing of uncertain demands," Optimization Eng. 6, 283-313 (2005).

[5] C.-S. Chang, D.-S. Lee, and Y.-S. Jou, "Load balanced Birkhoff-von Neumann switches. Part I. One-stage buffering," presented at the 2001 IEEE Workshop on High Performance Switching and Routing (HPSR), Kobe, Japan, 26-29 May 2001.

[6] C. Chekuri, G. Oriolo, M. G. Scutella, and F. B. Shepherd, "Hardness of robust network design," presented at the International Network Optimization Conference, Lisbon, Portugal, March 2005.

[7] N. G. Duffield, P. Goyal, A. Greenberg, P. Mishra, K. K. Ramakrishnan, and J. E. van der Merwe, "Resource management with hoses: point-to-cloud services for virtual private networks," IEEE/ACM Trans. Netw. 10, 679-692 (2002).

[8] F. Eisenbrand and F. Grandoni, "An improved approximation algorithm for virtual private network design," presented at the 16th Annual Association for Computing Machinery-Society for Industrial and Applied Mathematics Symposium On Discrete Algorithms, Vancouver, British Columbia, Canada, 23-25 January 2005.

[9] T. Erlebach and M. Ruegg, "Optimal bandwidth reservation in hose-model VPNs with multipath routing," in IEEE INFOCOM 2004 (IEEE Computer Society, 2004), Vol. 4, pp. 2275-2282.

[10] J. A. Fingerhut, S. Suri, and J. Turner, "Designing least-cost nonblocking broadband networks," J. Algorithms 22, 287-309 (1997).

[11] A. Gupta, J. Kleinberg, A. Kumar, R. Rastogi, and B. Yener, "Provisioning a virtual private network: a network design problem for multicommodity flow," presented at the 33rd Annual ACM Symposium on Theory of Computing, Hersonissos, Crete, Greece, July 2001.

[12] A. Gupta, A. Kumar, and T. Roughgarden, "Simpler and better approximation algorithms for network design," presented at the 35th Annual ACM Symposium on Theory of Computing, San Diego, California, 9-11 June 2003.

[13] C. A. J. Hurkens, J. C. M. Keijsper, and L. Stougie, "Virtual private network design: a proof of the tree routing conjecture on ring networks," in Proceedings of the 11th International Integer Programming and Combinatorial Optimization Conference, Vol. 3509 of Lecture Notes in Computer Science (Springer-Verlag, 2005), pp. 407-421.

[14] G. Italiano, S. Leonardi, and G. Oriolo, "Design of networks in the hose model," in Proceedings of 3 rd Workshop on Approximation and Randomization Algorithms in Communication Networks (ARACNE) (Carleton Scientific, 2002), pp. 65-76.

[15] I. Keslassy, S.-T. Chuang, K. Yu, D. Miller, M. Horowitz, O. Solgaard, and N. McKeown, "Scaling Internet routers using optics," in Proceedings of the ACM SIGCOMM 2003 Conference on Applications, Technologies, Architectures and Protocols for Computer Communications (Association for Computing Machinery, 2003), pp. 189-200.

[16] M. Kodialam, T. V. Lakshman, and S. Sengupta, "Efficient and robust routing of highly variable traffic," presented at HotNets III, San Diego, California, November 2004.

[17] T. Leighton and S. Rao, "An approximate max-flow min-cut theorem for uniform multicommodity flow problems with applications to approximation algorithms," in Proceedings of the 29th IEEE Symposium on Foundations of Computer Science (IEEE, 1988), pp. 422-431.

[18] D. Leung and W. D. Grover, "Capacity planning of survivable mesh-based transport networks under demand uncertainty," Photon. Netw. Commun. 10, 123-140 (2005).

[19] D. Mitra and R. A. Cieslak, "Randomized parallel communications on an extension of the omega network," J. Assoc. Comput. Mach. 32, 802-824 (1987).

[20] H. Nagesh, V. Poosala, V. Kumar, P. J. Winzer, and M. Zirngibl, "Load-balanced architecture for dynamic traffic," presented at the Optical Fiber Communication Conference, Anaheim, California, March 2005, paper OME67.

[21] H. Nagesh, V. Poosala, S. Sengupta, M. Alicherry, and V. Kumar, "NetSwitch: load-balanced data-over-optical architecture for mesh networks," Lucent Technical Memorandum ITD-0445867F (Lucent Technologies, 2004).

[22] A. Kumar, R. Rastogi, A. Silberschatz, and B. Yener, "Algorithms for provisioning virtual pri- 
vate networks in the hose model," IEEE/ACM Trans. Netw. 10, 565-578 (2002).

[23] H. Räcke, "Minimizing congestion in general networks, "in Proceedings of the 43rd Annual IEEE Symposium on Foundations of Computer Science (IEEE, 2002), pp. 43-52.

[24] S. Sengupta, V. Kumar, and D. Saha, "Switched optical backbone for cost-effective scalable core IP networks," IEEE Commun. Mag. 41(6) 60-70 (2003).

[25] L. G. Valiant, “A scheme for fast parallel communication,” SIAM J. Comput. 11(2), 350-361 (1982).

[26] I. Widjaja and A. I. Elwalid, "Exploiting parallelism to boost data-path rate in high-speed IP/MPLS networking," presented at the IEEE Workshop on High-Speed Networking, New York, New York, 23 June 2002.

[27] P. J. Winzer, F. B. Shepherd, P. Oswald, and M. Zirngibl, "Robust network design and selective randomized load balancing," presented at the European Conference on Optical Communications (ECOC'05), Glasgow, Scotland, 25-29 September 2005.

[28] R. Zhang-Shen and N. McKeown, "Designing a predictable Internet backbone network," presented at HotNets III, San Diego, California, November 2004.

[29] "Definitions and terminology for automatically switched optical networks (ASON)," ITU-T Recommendation G.8081 (International Telecommunication Union, 2004). 